UDK $577.1: 61$

ISSN 1452-8258

J Med Biochem 38: 71-82, 2019

Original paper

Originalni naučni rad

\title{
BIOCHEMICAL MARKERS FOR PREDICTION OF HYPERTENSIVE DISORDERS OF PREGNANCY
}

\author{
BIOHEMIJSKI MARKERI ZA PREDIKCIJU HIPERTENZIVNIH POREMEĆAJA U TRUDNOĆI
}

\author{
Dušica Kocijančić Belovic 1 , Snežana Plešinac 1,2, Jelena Dotlić1,2, Ana Savić Radojević ${ }^{2,3}$, \\ Slavica Akšam 1, Mirjana Marjanović Cvjetićanin ${ }^{1}$, Aleksandar Kocijančić4 \\ ${ }^{1}$ Clinic of Obstetrics and Gynecology, Clinical Center of Serbia, Belgrade, Serbia \\ ${ }^{2}$ Medical Faculty, University of Belgrade, Belgrade, Serbia \\ 3 Institute of Medical Biochemistry, Medical Faculty, University of Belgrade, Serbia \\ ${ }^{4}$ Clinic of Cardiology, Clinical Center of Serbia
}

\section{Summary}

Background: Gestational hypertension $(\mathrm{GH})$ and preeclampsia (PE) are the most common gestational complications. Several placental biochemical markers are used to predict $\mathrm{GH} / \mathrm{PE}$, but with conflicting results.

Methods: The study aim was to estimate the biochemical markers' ability to predict hypertensive disorders in pregnancy. On the first ultrasonographic examination, 104 healthy pregnant women were recruited. At the regular pregnancy check-ups, BMI, blood pressure, occurrence of gestational hypertension (early or late onset), preeclampsia, eclampsia and other complications were recorded. Serum concentrations (in multiples of median - MoM) of human chorionic gonadotropin (HCG) and pregnancyassociated plasma protein A (PAPPA) were measured from the 11th to 14th gestational week, while HCG, alpha feto protein (AFP), estriol and inhibin were determined between the 16th and 19th gestational week.

Results: Hypertensive disorders throughout pregnancy were diagnosed in $20.2 \%$ women. Early-onset $\mathrm{GH}$ was registered in 7 and PE in 6 patients, while 14 had late-onset GH and 10 additional women PE. There were no significant differences $(p \geq 0.05)$ in biochemical markers concentrations between women with and without GH/PE. PAPPA levels in the first and HCG in the second trimester correlated with early and late GH/PE. Moreover, higher AFP concentra-

\section{Kratak sadržaj}

Uvod: Gestacijska hipertenzija (GH) i preeklampsija (PE) najčešće su gestacijske komplikacije. Nekoliko placentnih biohemijskih markera koristi se za predikciju GH/PE, ali sa oprečnim rezultatima.

Metode: Cilj studije bila je procena mogućnosti korišćenja biohemijskih markera za predikciju hipertenzivnih poremećaja u trudnoći. Na prvom ultrasonografskom pregledu, u studiju su uvrštene 104 zdrave trudnice. Na redovnim pregledima tokom trudnoće registrovani su ITM, krvni pritisak, pojava gestacijske hipertenzije (rani ili kasni početak), preeklampsija, eklampsija i druge komplikacije. Serumske koncentracije (izražene u umnošcima medijane - MOM) humanog horionskog gonadotropina (HCG) i plazma proteina vezanog za trudnoću (PAPPA) merene su u periodu od 11. do 14. gestacijske nedelje, dok su HCG, alfa feto protein (AFP), estriol i inhibin određivani između 16. i 19. nedelje.

Rezultati: Hipertenzivni poremećaji tokom trudnoće dijagnostikovani su kod 20,2\% žena. Rana gestacijska hipertenzija registrovana je kod 7, a preeklampsija kod 6 pacijentkinja, dok je kasnu gestacijsku hipertenziju imalo 14, a preeklampsiju 10 žena. Nije bilo statistički značajne razlike $(p>0,05)$ u vrednostima biohemijskih markera kod pacijentkinja sa ili bez GH/PE. Vrednosti PAPPA u prvom i HCG-a u drugom trimestru korelirale su sa pojavom rane i kasne GH/PE. S

Address for correspondence:

Dusica Kocijancic, MD, Ob/Gyn

Clinic of Obstetrics and Gynecology,

Clinical Center of Serbia

Koste Todorovica 26, 11000 Belgrade, Serbia

Telephone: +381113615592

e-mail: dusicakocijancic@yahoo.com 
tions were registered in women with preeclampsia signs/symptoms. According to ROC analysis, AFP $>1.05$ MoM properly identified $80 \%$ of $\mathrm{GH} / \mathrm{PE}$ cases. Obtained models imply that HCG, PAPPA and AFP should be used for $\mathrm{GH} / \mathrm{PE}$ prediction.

Conclusions: Biochemical markers HCG, PAPPA and AFP could be useful in predicting gestational hypertension and preeclampsia. However, different markers should be used for early and late onset GH/PE.

Keywords: gestational hypertension, preeclampsia, HCG, AFP, estriol, inhibin

\section{Introduction}

During physiological gestation, the placenta releases a number of factors into the maternal circulation that are involved in the general regulation of maternal metabolism during pregnancy, inducing physiological adaptations required for successful pregnancy, as well as fetal growth and development $(1,2)$. These compounds can be found in maternal blood and are used as biochemical pregnancy markers. Their serum concentrations can be altered (increased or decreased) in different pathological conditions during pregnancy (3). Therefore, as their changes are associated in a unique manner to the pregnancy disorder in question, numerous studies have investigated the potential role of biochemical pregnancy markers in the diagnosis and early prediction of gestational complications (4).

Pregnancy complications mostly develop in the second half of gestation, but are thought to be caused by different pathological mechanisms in the first weeks of pregnancy (1). Numerous gestational illnesses share similar etiology which is based on dysfunctional trophoblast invasion and placental formation. Early identification of high-risk pregnancies may facilitate antenatal surveillance or prevention and consequently improve pregnancy outcome (2).

Among the most important gestational complications are hypertensive disorders of pregnancy, which occur in approximately 2 and $10 \%$ of pregnancies and can cause significant morbidity and mortality of both mothers and children $(5,6)$. Preeclampsia affects around $3 \%$ of pregnancies, but is difficult to predict as the onset and severity of disease are unpredictable $(7,8)$. The clinical syndrome of hypertensive disorders of pregnancy mostly develops in the third pregnancy trimester, but in some women can be apparent in the first half of pregnancy, which is a more severe condition $(4,3)$. Many underlying factors causing gestational hypertension and preeclampsia actually already exist from early weeks of gestation in many patients. However, there is currently no reliable screening method in the first trimester of pregnancy with sufficient accuracy to identify women at high risk of developing gestational hypertension $(\mathrm{GH})$ or preeclampsia $(\mathrm{PE})(1,2)$. druge strane, kod pacijentkinja sa znacima i simptomima preeklampsije registrovane su povišene koncentracije AFPa. Prema ROC analizi, AFP > 1,05 MoM precizno identifikuje $80 \%$ slučajeva GH/PE. Dobijeni modeli podrazumevaju da HCG, PAPPA i AFP treba koristiti za predviđanje $\mathrm{GH} / \mathrm{PE}$

Zaključak: Biohemijski markeri HCG, PAPPA i AFP mogu biti korisni u predikciji gestacijske hipertenzije i preeklampsije. Ipak, za GH/PE sa ranim i kasnim početkom trebalo bi koristiti različite markere.

Ključne reči: gestacijska hipertenzija, preeklampsija, HCG, AFP, estriol, inhibin

Several biochemical markers measured in the first and second pregnancy trimester have been used to predict occurrence of GH/PE in the later course of pregnancy $(9,10)$. Still, although different biomarkers of placental function and vascularisation have been associated with $\mathrm{GH} / \mathrm{PE}$ occurrence in the literature data, few are used diagnostically in routine clinical settings $(1,3)$. The most commonly assessed in daily practice are those biochemical markers that are already proven as predictors of genetic aberrations and adverse pregnancy outcomes incorporated in first and second trimester screening (4). However, heterogeneous results from different studies have been obtained and therefore, relevance of placental biomarkers is not still defined $(11,12)$. Further research should clarify the reliability and usefulness of these biochemical markers for GH/PE prediction.

The aim of our study was to estimate the ability of biochemical markers of the first and second trimesters to predict hypertensive disorders of pregnancy.

\section{Meterial and Methods}

Study included all consecutive pregnant women who had regular pregnancy check-ups in the Clinic of Obstetrics and Gynecology, Clinical Center of Serbia. Women were recruited for the study during a threemonth period (January 1 - March 31 2017) on the first ultrasonographic examination upon pregnancy confirmation. Exclusion criteria were having chronic illnesses and pregnancies conceived by assisted reproduction. All investigated patients signed informed consent for the study.

Detailed general medical (family and personal) and obstetrical history were taken from every patient regarding age, cigarette smoking status, method of conception, hereditary predisposition for hypertension, chronic illnesses, parity, gestational complications and outcomes of previous pregnancies (hypertension, diabetes, pregnancy loss, gestational week of delivery, Apgar score of the child).

Investigated women were regularly checked-up at least once per trimester in our Clinic. At every 
examination, we measured the patient's height and weight and calculated their BMI, took a blood and 24-hour urine sample for classic laboratory testing (blood count, coagulation factors, glucose blood concentration, AST, ALT, LDH, proteinuria, creatinine, urea). All pregnancies were dated by last menstrual period and ultrasonographic crown-rump length $(C R L)$ measurements performed in the first trimester.

In the first trimester (11 to 14 gestational weeks) biochemical markers that are incorporated in Double test screening such as beta subunit of human chorionic gonadotropin (beta subunit of HCG) and pregnancy-associated plasma protein A (PAPPA) were assessed, while in the second trimester (16 to 19 gestational weeks) study authors evaluated markers used for Triple testing i.e. beta HCG, alpha fetoprotein $(\mathrm{AFP})$, estriol (E3) and inhibin (INH). Approximately 5 to 10 milliliters of blood was drawn by venipuncture into nonheparinized tubes and centrifuged for 15 minutes. After serum separation, concentrations of the investigated biochemical markers were measured by using a BRAHMS KRYPTOR analyzer and applying fluorocytometric immunoassay with SsdwLab 5 software. The measured serum concentrations (IU/L) of biomarkers were then converted into multiples of median (MoM) and adjusted for gestational week for easier comparison and analysis. The standard referral range of all tested pregnancy markers, most widely used in literature and adopted in this study as well, is from 0.5 to 2 MoM.

Every patient regularly measured blood pressure and reported potential signs and symptoms of preeclampsia/eclampsia (headache, epigastric pain, edema of extremities, impaired vision, convulsions). As study outcomes, authors considered gestational hypertension $(\mathrm{GH})$, preeclampsia $(\mathrm{PE})$ and eclampsia (E). In this study, authors used the currently widely accepted definitions of investigated conditions (ACOG). Gestational hypertension is defined as blood pressure 140/90 $\mathrm{mmHg}$ measured 2 times after $20^{\text {th }}$ gestational week in a woman with previously normal blood pressure. If this condition is associated with coexisting significant proteinuria $(300 \mathrm{mg}$ in a $24 \mathrm{~h}$ urine specimen) it is defined as preeclampsia and if convulsions occur, eclampsia is diagnosed. $\mathrm{GH}$ and PE can be further subdivided according to the time of diagnosis into early-onset (starting before 34 weeks of gestation) and late-onset (developing after 34 weeks of gestation). Study authors tested the predictive value of biochemical markers for GH/PR concerning its onset as well as throughout pregnancy (regardless of diagnosis time).

Study authors recorded all adverse pregnancy outcomes (miscarriages i.e. spontaneous abortions before $24^{\text {th }}$ gestational week, intrauterine fetal death, as well as gestational complications that could be related with hypertension like placental abruption and HELLP syndrome). Finally, upon birth, study authors noted the birth-weight and Apgar score of the child, as well as the gestational week (GW) of delivery (prematurity was considered if delivery occurred before the $37^{\text {th }} \mathrm{GW}$ ).

\section{Statistics}

Data were analyzed using methods of descriptive (number, percent, mean, standard deviation) and analytical statistics and applying the SPSS 20 software. Correlations of biochemical parameters and occurrence of hypertension were tested using Spearman correlation. Significance of differences between categories of assessed parameters as well as between women with and without hypertension in pregnancy was examined by $\chi^{2}$ test and Kruscal Wallis nonparametric ANOVA.

Study authors performed the ROC (Receiver Operating Characteristics) analysis to set the cut-off values of biochemical parameters that could imply development of hypertension in pregnancy.

Finally, Enter and Forward Wald binary logistic regression were applied to construct models for prediction of hypertension in pregnancy occurrence based on investigated biochemical markers. All models were adjusted for potential confoundings (mothers' age, $\mathrm{BMI}$ and parity). However, no significant confoundings were found in the obtained models.

\section{Results}

Study included 104 pregnant women that on average had $30.54+/-4.93$ years of age and adequate BMI mean+/-sd 28.30+/-2.21 throughout pregnancy. Significantly more investigated women were not smokers (Table I). They had up to four previous pregnancies which have mostly in $91.3 \%$ been uneventful ending in term with a healthy child. In our sample, $75 \%$ of women did not have positive family history of hypertension, while only four women had gestational hypertension in previous pregnancy (Table I).

In the assessed pregnancy study, authors registered two spontaneous abortions in the second trimester and one case of intrauterine fetal death in the $30^{\text {th }}$ gestational week. There were seven cases of placental abruption (Table I). The mean+/-sd gestational week at the time of delivery in our study was 36.94+/-6.25. Still, the majority of children had good birth-weight (mean+/-sd = 3120.84+/-809.12 grams) and Apgar score (mean+/-sd=8.22+/-1.62).

There were seven patients diagnosed with earlyonset gestational hypertension, while 14 more patients developed hypertension after the $34^{\text {th }}$ gestational week (all together $20.2 \%$ of women). Moreover, six patients had preeclampsia symptoms and signs already in the second trimester while ten more were diagnosed with preeclampsia in the third trimester 
74 Kocijancic Belovic et al.: Markers of pregnancy hypertension

Table I Frequency of history data related to hypertensive disorders as well as occurrence of hypertension in pregnancy.

\begin{tabular}{|c|c|c|c|c|c|}
\hline \multicolumn{2}{|l|}{ Parameters } & Frequency & Percent & $\chi^{2}$ & $\mathrm{p}$ \\
\hline \multirow[t]{2}{*}{ Early-onset GH } & yes & 7 & 6.7 & \multirow{2}{*}{74.941} & \multirow{2}{*}{0.001} \\
\hline & no & 97 & 93.3 & & \\
\hline \multirow[t]{2}{*}{ Late-onset GH } & yes & 14 & 14.4 & \multirow{2}{*}{33.640} & \multirow{2}{*}{0.001} \\
\hline & no & 83 & 85.6 & & \\
\hline \multirow[t]{4}{*}{ Preeclampsia symptoms } & headache & 5 & 4.8 & \multirow{4}{*}{56.980} & \multirow{4}{*}{0.001} \\
\hline & edema & 2 & 1.9 & & \\
\hline & combined & 9 & 8.7 & & \\
\hline & no & 88 & 84.6 & & \\
\hline \multirow[t]{2}{*}{ Eclampsia } & yes & 0 & 0.0 & \multirow{2}{*}{99.999} & \multirow{2}{*}{0.001} \\
\hline & no & 104 & 100.0 & & \\
\hline \multirow[t]{2}{*}{ HELLP Sy in the third trimester } & yes & 1 & 0.9 & \multirow{2}{*}{96.040} & \multirow{2}{*}{0.001} \\
\hline & no & 103 & 99.1 & & \\
\hline \multirow[t]{3}{*}{ Placental abruption } & in pregnancy & 2 & 1.9 & \multirow{3}{*}{162.317} & \multirow{3}{*}{0.001} \\
\hline & in delivery & 5 & 4.8 & & \\
\hline & no & 97 & 93.3 & & \\
\hline \multirow[t]{2}{*}{ Smoking status } & yes & 36 & 34.6 & \multirow{2}{*}{9.846} & \multirow{2}{*}{0.002} \\
\hline & no & 68 & 65.4 & & \\
\hline \multirow[t]{2}{*}{ HTA in family } & yes & 25 & 24.0 & \multirow{2}{*}{89.558} & \multirow{2}{*}{0.001} \\
\hline & no & 79 & 75.9 & & \\
\hline \multirow[t]{2}{*}{ HTA in previous pregnancy } & yes & 5 & 4.8 & \multirow{2}{*}{84.962} & \multirow{2}{*}{0.001} \\
\hline & no & 99 & 95.2 & & \\
\hline \multirow[t]{3}{*}{ Proteinuria in II trimester } & 0.3 to $2 \mathrm{~g}$ & 5 & 4.8 & \multirow{3}{*}{93.158} & \\
\hline & above $2 \mathrm{~g}$ & 0 & 0 & & 0.001 \\
\hline & no & 99 & 95.2 & & \\
\hline Proteinuria in III trimester & 0.3 to $2 \mathrm{~g}$ & 12 & 11.5 & & \\
\hline & above $2 \mathrm{~g}$ & 4 & 3.8 & 144.740 & 0.001 \\
\hline & no & 88 & 84.7 & & \\
\hline Oliguria in II trimester & yes & 104 & 100.0 & 99999 & 1חمח ח \\
\hline & no & 0 & 0.0 & 99.999 & 0.001 \\
\hline Oliguria in III trimester & yes & 100 & 96.2 & 5 & 1חمח ח \\
\hline & no & 4 & 3.8 & 95.005 & 0.001 \\
\hline
\end{tabular}

Legend: GH - gestational hypertension; PE - preeclampsia; HTA - hypertension

(15.4\% of women throughout pregnancy). There were no cases of eclampsia in our sample, but one patient developed HELLP syndrome in the third trimester (Table I).

The findings of biochemical markers of the first and second pregnancy trimester are presented in
Table II and III. Majority of patients had all markers in the referral range throughout pregnancy. There were no significant differences $(p \geq 0.05)$ in mean serum concentrations (in MoM) of biochemical markers between women with and without hypertension in pregnancy. 
Table II Serum concentrations of investigated biochemical pregnancy markers and standard laboratory tests that could imply hypertension in pregnancy.

\begin{tabular}{|c|c|c|c|c|c|c|c|}
\hline \multirow{2}{*}{ Parameters } & & \multicolumn{2}{|c|}{ Whole sample } & \multicolumn{2}{|c|}{$\mathrm{GH} / \mathrm{PE}$ group } & \multicolumn{2}{|c|}{ Healthy women } \\
\hline & & Mean & SD & Mean & SD & Mean & SD \\
\hline \multirow{2}{*}{$\begin{array}{l}\text { I trimester } \\
\text { (in MoM) }\end{array}$} & $\beta \mathrm{HCG}(\mathrm{mlU} / \mathrm{mL})$ & 1.27 & 0.92 & 1.31 & 1.28 & 1.26 & 0.82 \\
\hline & PAPPA (mlU/mL) & 1.48 & 1.08 & 1.10 & 0.99 & 1.59 & 1.09 \\
\hline \multirow{4}{*}{$\begin{array}{l}\text { II trimester } \\
\text { (in MoM) }\end{array}$} & $\beta$ HCG $(\mathrm{mlU} / \mathrm{mL})$ & 1.71 & 1.02 & 1.18 & 0.91 & 1.93 & 1.01 \\
\hline & $\operatorname{AFP}(\mathrm{ng} / \mathrm{mL})$ & 1.22 & 0.59 & 1.17 & 0.24 & 1.23 & 0.68 \\
\hline & E3 (ng/mL) & 1.18 & 0.62 & 0.96 & 0.24 & 1.26 & 0.71 \\
\hline & Inhibin (pg/mL) & 1.21 & 0.49 & 1.23 & 0.11 & 1.17 & 0.98 \\
\hline \multirow{5}{*}{ I trimester } & AST (U/L) & 16.40 & 1.91 & 17.35 & 2.21 & 14.15 & 1.05 \\
\hline & $\mathrm{ALT}(\mathrm{U} / \mathrm{L})$ & 11.58 & 0.83 & 9.89 & 0.98 & 13.03 & 1.47 \\
\hline & LDH (U/L) & 287.10 & 15.25 & 312.27 & 34.19 & 274.63 & 22.26 \\
\hline & Urea (mmol/L) & 3.69 & 0.41 & 3.51 & 0.29 & 3.73 & 0.37 \\
\hline & Creatinine $(\mu \mathrm{mol} / \mathrm{L})$ & 60.45 & 4.56 & 63.56 & 7.89 & 58.22 & 4.45 \\
\hline \multirow{5}{*}{ II trimester } & AST (U/L) & 17.27 & 1.11 & 18.00 & 2.95 & 16.37 & 1.05 \\
\hline & $\operatorname{ALT}(\mathrm{U} / \mathrm{L})$ & 12.15 & 3.60 & 11.49 & 3.28 & 14.10 & 3.38 \\
\hline & LDH (U/L) & 328.01 & 21.71 & 336.55 & 18.50 & 293.61 & 28.12 \\
\hline & Urea $(\mathrm{mmol} / \mathrm{L})$ & 3.41 & 0.14 & 3.77 & 0.56 & 2.90 & 0.22 \\
\hline & Creatinine $(\mu \mathrm{mol} / \mathrm{L})$ & 64.74 & 6.61 & 64.12 & 8.04 & 62.28 & 5.52 \\
\hline
\end{tabular}

Legend: $\beta$ HCG - Beta subunit of human chorionic gonadotropin; PAPPA - pregnancy-associated plasma protein A; AFP alpha feto protein; E3 - estriol; ALT - alanine transaminase; AST - aspartate transaminase; LDH - lactate dehydrogenase; MoM - multiples of median.

Serum levels of PAPPA in the first and HCG in the second trimester of pregnancy positively correlated with occurrence of both early and late gestational hypertension as well as placental abruption during pregnancy. Moreover, higher concentrations of AFP were registered in women who presented with signs and symptoms of preeclampsia (Table IV). There were no significant correlations between biochemical markers of the first and second pregnancy trimester and standard biochemical laboratory tests measured throughout the pregnancy as well as history data that could imply on gestational HTA/PE (Table IV). Moreover, no significant correlations of pregnancy biochemical markers and blood coagulation factors were found, except for antithrombin III $(\rho=0.226$; $p=0.026$ ) that was increased above the referral preg- nancy range in patients with higher first trimester serum levels of HCG and PAPPA.

Study authors constructed significant models for predicting occurrence of early-onset hypertension based on only first trimester markers $\left(\chi^{2}=18.840\right.$; $\mathrm{p}=0.016 ; \quad \mathrm{B}=2.543 ;$ Wald $=41.958 ;$ Nagelkerke $\mathrm{R}^{2}=0.616$; total classification $\left.=90.6 \%\right)$ and all biochemical markers together $\left(\chi^{2}=15.844 ; \mathrm{p}=0.017\right.$; $\mathrm{B}=1.540$; Wald $=5.863$; Nagelkerke $\mathrm{R}^{2}=0.998$; total classification $=98.1 \%)$. For markers of the second trimester, the model was not significant $(p=0.107)$.

Significant logistic regression equations were achieved also for development of late-onset hypertension when authors tested biochemical markers of only second trimester $\left(\chi^{2}=13.666 ; \mathrm{p}=0.018 ; \mathrm{B}=0.956\right.$; Wald =3.297; Nagelkerke $R^{2}=0.767$; total classifica- 
Table III Categories of tested biochemical markers of pregnancy in investigated women.

\begin{tabular}{|c|c|c|c|c|c|c|c|}
\hline & arameters & Wh & nple & $p$ between & $\mathrm{GH} / \mathrm{PE}$ group & $\begin{array}{l}\text { Healthy } \\
\text { controls }\end{array}$ & Between \\
\hline & & No & $\%$ & & $\mathrm{No}=21$ & $\mathrm{No}=83$ & \\
\hline & below $0.5 \mathrm{MoM}$ & 14 & 13.5 & & 6 & 8 & \\
\hline categories I & from 0.5 to $2 \mathrm{MoM}$ & 74 & 71.2 & 0.001 & 12 & 62 & 0.127 \\
\hline & over $2 \mathrm{MoM}$ & 16 & 15.3 & & 3 & 13 & \\
\hline & below $0.5 \mathrm{MoM}$ & 13 & 12.5 & & 6 & 7 & \\
\hline $\begin{array}{l}\text { PAPPA } \\
\text { categories }\end{array}$ & from 0.5 to $2 \mathrm{MoM}$ & 66 & 63.5 & 0.001 & 12 & 54 & 0.032 \\
\hline & over $2 \mathrm{MoM}$ & 25 & 24.0 & & 3 & 22 & \\
\hline & below $0.5 \mathrm{MoM}$ & 10 & 9.6 & & 8 & 2 & \\
\hline categories II & from 0.5 to $2 \mathrm{MoM}$ & 75 & 72.2 & 0.001 & 10 & 65 & 0.003 \\
\hline & over $2 \mathrm{MoM}$ & 19 & 18.3 & & 3 & 16 & \\
\hline & below $0.5 \mathrm{MoM}$ & 26 & 25.0 & & 2 & 24 & \\
\hline $\begin{array}{l}\text { AFP } \\
\text { categories }\end{array}$ & from 0.5 to $2 \mathrm{MoM}$ & 49 & 47.1 & 0.011 & 13 & 36 & 0.265 \\
\hline & over $2 \mathrm{MoM}$ & 29 & 27.9 & & 6 & 23 & \\
\hline & below $0.5 \mathrm{MoM}$ & 14 & 11.5 & & 4 & 10 & \\
\hline $\begin{array}{l}\text { E3 } \\
\text { categories }\end{array}$ & from 0.5 to $2 \mathrm{MoM}$ & 78 & 77.0 & 0.001 & 13 & 65 & 0.856 \\
\hline & over 2 MoM & 12 & 11.5 & & 4 & 8 & \\
\hline & below $0.5 \mathrm{MoM}$ & 0 & 0 & & 0 & 0 & \\
\hline $\begin{array}{l}\text { Inhibin } \\
\text { categories }\end{array}$ & from 0.5 to $2 \mathrm{MoM}$ & 104 & 100 & 0.001 & 21 & 83 & 0.001 \\
\hline & over 2 MoM & 0 & 0 & & 0 & 0 & \\
\hline
\end{tabular}

Legend: No - number; $\mathrm{p}$ - statistical significance; Bold - significant; HCG - human chorionic gonadotropin; PAPPA - pregnancy-associated plasma protein A; AFP - alpha feto protein; E3 - estriol; MoM - multiples of median.

tion $=88.9 \%)$ and all biochemical markers together $\left(\chi^{2}=20.597 ; \mathrm{p}=0.004 ; \quad \mathrm{B}=0.875 ;\right.$ Wald $=2.705$; Nagelkerke $\mathrm{R}^{2}=0.998$; total classification $=89.8 \%$ ). For markers of the first trimester the model was not significant $(p=0.184)$.

Finally, study authors obtained significant models for predicting occurrence of hypertension throughout pregnancy (early and/or late onset) based on only first trimester markers $\left(\chi^{2}=16.385\right.$; $\mathrm{p}=0.017$; $\mathrm{B}=1.299$; Wald $=7.854 ;$ Nagelkerke $\mathrm{R}^{2}=0.698$; total classification $\left.=78.6 \%\right)$, only second trimester markers $\left(\chi^{2}=24.112 ; \mathrm{p}=0.001 ; \mathrm{B}=0.946\right.$; Wald $=0.751$; Nagelkerke $\mathrm{R}^{2}=0.672$; total classification $=88.9 \%$ ) and all biochemical markers together $\left(\chi^{2}=18.556 ; \quad p=0.015 ; \quad B=2.875 ; \quad W a l d=4.705\right.$; Nagelkerke $\mathrm{R}^{2}=0.972$; total classification $=96.5 \%$ ).
Obtained models are presented in the Table $\mathrm{V}$ while results of ROC analysis are presented in Table VI and Figure 1. According to the performed ROC analysis, the only biochemical marker that could be used for prediction of hypertensive disorders during pregnancy was AFP measured between the $16^{\text {th }}$ and $19^{\text {th }}$ gestational week. If AFP was higher than $1.05 \mathrm{MoM}$ then $80 \%$ of women that would develop GH/PE in further pregnancy course would be properly identified. Authors in this study did not manage to prove the reliability of biochemical markers for prediction of early-onset GH/PE. Moreover, the same ROC curve was obtained for late-onset $\mathrm{GH} / \mathrm{PE}$ or its occurrence throughout pregnancy regardless of onset time. 
Table IV Correlations of biochemical markers (concentrations in MoM) and occurrence of gestational hypertensive disorders and parameters that imply GH/PE.

\begin{tabular}{|c|c|c|c|c|c|c|c|}
\hline \multirow{2}{*}{\multicolumn{2}{|c|}{ Parameters }} & \multicolumn{2}{|c|}{ I trimester } & \multicolumn{4}{|c|}{ II trimester } \\
\hline & & \multirow{2}{*}{$\frac{\beta H C G}{0.228}$} & \multirow{2}{*}{$\begin{array}{c}\text { PAPPA } \\
\mathbf{0 . 5 1 6}\end{array}$} & \multirow{2}{*}{$\frac{\beta \text { HCG }}{0.007}$} & \multirow{2}{*}{$\begin{array}{c}\text { AFP } \\
0.216\end{array}$} & \multirow{2}{*}{$\frac{E 3}{-0.775}$} & \multirow{2}{*}{$\begin{array}{l}\text { Inhibin } \\
-0.019\end{array}$} \\
\hline Early-onset GH & $\rho$ & & & & & & \\
\hline & $\mathrm{p}$ & 0.025 & 0.028 & 0.946 & 0.390 & 0.225 & 0.852 \\
\hline \multirow[t]{2}{*}{ Late-onset GH } & $\rho$ & 0.269 & 0.465 & -0.131 & 0.167 & -0.775 & 0.071 \\
\hline & $\mathrm{p}$ & 0.008 & 0.045 & 0.603 & 0.507 & 0.225 & 0.492 \\
\hline \multirow[t]{2}{*}{ Early-onset PE } & $\rho$ & -0.027 & -0.082 & 0.979 & 0.229 & -0.211 & -0.007 \\
\hline & $\mathrm{p}$ & 0.797 & 0.732 & 0.041 & 0.360 & 0.789 & 0.945 \\
\hline \multirow[t]{2}{*}{ Late-onset PE } & $\rho$ & -0.133 & -0.212 & 0.184 & -0.014 & 0.894 & 0.056 \\
\hline & $\mathrm{p}$ & 0.195 & 0.383 & 0.464 & 0.957 & 0.106 & 0.588 \\
\hline \multirow{2}{*}{$\begin{array}{l}\text { HELLP syndrome } \\
\text { in the III trimester }\end{array}$} & $\rho$ & 0.143 & 0.344 & 0.023 & -0.023 & -0.143 & -0.060 \\
\hline & $\mathrm{p}$ & 0.166 & 0.149 & 0.927 & 0.927 & 0.197 & 0.558 \\
\hline \multirow[t]{2}{*}{ Placental abruption } & $\rho$ & 0.214 & 0.473 & 0.020 & -0.174 & -0.219 & 0.113 \\
\hline & $\mathrm{p}$ & 0.036 & 0.035 & 0.936 & 0.490 & 0.482 & 0.269 \\
\hline \multirow[t]{2}{*}{ Age } & $\rho$ & 0.007 & 0.012 & 0.440 & -0.049 & -0.239 & -0.700 \\
\hline & $\mathrm{p}$ & 0.946 & 0.910 & 0.052 & 0.842 & 0.340 & 0.188 \\
\hline \multirow[t]{2}{*}{ Body Mass Index } & $\rho$ & -0.065 & 0.007 & -0.027 & -0.016 & 0.333 & 0.003 \\
\hline & $\mathrm{p}$ & 0.519 & 0.946 & 0.911 & 0.947 & 0.177 & 0.987 \\
\hline \multirow[t]{2}{*}{ Smoking status } & $\rho$ & 0.064 & 0.036 & -0.248 & 0.229 & 0.011 & 0.007 \\
\hline & $\mathrm{p}$ & 0.525 & 0.724 & 0.292 & 0.345 & 0.965 & 0.946 \\
\hline \multirow[t]{2}{*}{ HTA in family } & $\rho$ & 0.153 & 0.077 & 0.152 & -0.200 & 0.244 & 0.005 \\
\hline & $\mathrm{p}$ & 0.129 & 0.449 & 0.523 & 0.411 & 0.328 & 0.967 \\
\hline \multirow{2}{*}{$\begin{array}{l}\mathrm{GH} / \mathrm{PE} \\
\text { in previous pregnancy }\end{array}$} & $\rho$ & -0.179 & -0.167 & -0.100 & -0.043 & 0.210 & -0.065 \\
\hline & $\mathrm{p}$ & 0.075 & 0.100 & 0.676 & 0.861 & 0.402 & 0.519 \\
\hline \multirow{2}{*}{$\begin{array}{l}\text { AST } \\
\text { (mean I or II trimester acc.) }\end{array}$} & $\rho$ & -0.164 & -0.150 & -0.259 & -0.301 & -0.398 & 0.005 \\
\hline & $\mathrm{p}$ & 0.108 & 0.145 & 0.271 & 0.210 & 0.102 & 0.967 \\
\hline \multirow{2}{*}{$\begin{array}{l}\text { ALT } \\
\text { (mean I or II trimester acc.) }\end{array}$} & $\rho$ & -0.037 & 0.142 & 0.224 & -0.129 & -0.158 & 0.258 \\
\hline & $\mathrm{p}$ & 0.716 & 0.167 & 0.356 & 0.609 & 0.531 & 0.742 \\
\hline \multirow{2}{*}{$\begin{array}{l}\text { LDH } \\
\text { (mean I or II trimester acc.) }\end{array}$} & $\rho$ & -0.164 & -0.150 & -0.259 & -0.301 & -0.398 & 0.001 \\
\hline & $\mathrm{p}$ & 0.108 & 0.145 & 0.271 & 0.210 & 0.102 & 0.998 \\
\hline \multirow{2}{*}{$\begin{array}{l}\text { Urea } \\
\text { (mean I or II trimester acc.) }\end{array}$} & $\rho$ & -0.134 & 0.060 & -0.775 & -0.775 & 0.775 & -0.258 \\
\hline & $\mathrm{p}$ & 0.190 & 0.561 & 0.225 & 0.225 & 0.225 & 0.742 \\
\hline \multirow{2}{*}{$\begin{array}{l}\text { Creatinine } \\
\text { (mean I or II trimester acc) }\end{array}$} & $\rho$ & -0.047 & 0.131 & -0.258 & 0.258 & -0.775 & -0.775 \\
\hline & $\mathrm{p}$ & 0.645 & 0.202 & 0.742 & 0.742 & 0.225 & 0.225 \\
\hline \multirow[t]{2}{*}{ Oliguria (I or II trimester acc.) } & $\rho$ & -0.098 & -0.058 & -0.258 & 0.258 & -0.775 & -0.775 \\
\hline & $\mathrm{p}$ & 0.340 & 0.572 & 0.742 & 0.742 & 0.225 & 0.225 \\
\hline
\end{tabular}

Legend: Bold - significant; acc - accordingly; HCG - human chorionic gonadotropin; PAPPA - pregnancy-associated plasma protein A; AFP - alpha feto protein; E3 - estriol; ALT - alanine transaminase; AST - aspartate transaminase; LDH - lactate dehydrogenase; MoM - multiples of median; HTA - hypertension; GH - gestational hypertension; PE - preeclampsia. 
78 Kocijancic Belovic et al.: Markers of pregnancy hypertension

Table V Models for prediction of GH/PE during pregnancy based on biochemical markers serum concentrations (in MoM).

\begin{tabular}{|c|c|c|c|}
\hline Parameters & Early-onset GH/PE & Late-onset GH/PE & $\mathrm{GH} / \mathrm{PE}$ throughout pregnancy \\
\hline I trimester markers & $\begin{array}{l}1.576 \\
-0.798 \text { x HCG I trim } \\
+1.504 \text { x PAPPA }\end{array}$ & No significant model & $\begin{array}{l}-4.168 \\
-0.659 \text { x PAPPA }\end{array}$ \\
\hline II trimester markers & No significant model & $\begin{array}{l}70.419 \\
+5.891 \times \text { HCG II trim } \\
+4.379 \times \text { AFP }\end{array}$ & $\begin{array}{l}-70.419 \\
-4.379 \times \text { AFP }\end{array}$ \\
\hline All biochemical markers & $\begin{array}{l}-63.126 \\
-25.491 \times \text { HCG I trim } \\
+37.27 \times \text { HCG II trim } \\
+63.661 \times \text { AFP }\end{array}$ & $\begin{array}{l}-244.185 \\
-18.745 \times \text { HCG I trim } \\
+52.126 \times \text { HCG II trim } \\
+40.128 \times \text { AFP }\end{array}$ & $\begin{array}{l}244.185 \\
+18.745 \times \text {. HCG I trim } \\
-52.126 \times \text { HCG II trim } \\
-40.128 \times \text { AFP }\end{array}$ \\
\hline
\end{tabular}

Legend: trim - trimester; GH - gestational hypertension; PE - preeclampsia; HCG - human chorionic gonadotropin; PAPPA pregnancy-associated plasma protein A; AFP - alpha feto protein; E3 - estriol; MoM - multiples of median

Table VI ROC analysis for biochemical parameters (concentrations in MoM) that could imply occurrence of hypertensive disorder during pregnancy.

\begin{tabular}{|c|c|c|c|c|c|c|}
\hline \multicolumn{2}{|c|}{ Parameters in MoM } & AUC & $p$ & Cut-off value & Sensitivity \% & Specificity \% \\
\hline \multirow{5}{*}{ Early onset } & $\beta \mathrm{HCG}$ I trimester & 0.524 & 0.901 & 1.33 & 66.7 & 42.9 \\
\hline & PAPA I trimester & 0.357 & 0.450 & 0.56 & 66.7 & 44.3 \\
\hline & $\beta \mathrm{HCG}$ II trimester & 0.250 & 0.186 & 2.22 & 33.3 & 57.1 \\
\hline & AFP II trimester & 0.501 & 0.987 & 1.04 & 66.7 & 50.0 \\
\hline & E3 II trimester & 0.286 & 0.257 & 1.11 & 33.3 & 57.1 \\
\hline \multirow{5}{*}{ Late onset } & $\beta \mathrm{HCG}$ I trimester & 0.400 & 0.527 & 1.33 & 60.0 & 41.7 \\
\hline & PAPA I trimester & 0.350 & 0.343 & 2.73 & 40.0 & 75.0 \\
\hline & $\beta \mathrm{HCG}$ II trimester & 0.125 & 0.399 & 0.53 & 80.0 & 50.0 \\
\hline & AFP II trimester & 0.633 & 0.018 & 1.04 & 80.0 & 58.3 \\
\hline & E3 II trimester & 0.342 & 0.317 & 0.94 & 60.0 & 33.3 \\
\hline \multirow{5}{*}{$\begin{array}{l}\text { Throughout } \\
\text { pregnancy }\end{array}$} & $\beta \mathrm{HCG}$ I trimester & 0.400 & 0.527 & 1.33 & 60.0 & 41.7 \\
\hline & PAPA I trimester & 0.350 & 0.343 & 2.73 & 40.0 & 75.0 \\
\hline & $\beta \mathrm{HCG}$ II trimester & 0.125 & 0.399 & 2.20 & 20.0 & 50.0 \\
\hline & AFP II trimester & 0.633 & 0.018 & 1.05 & 80.0 & 58.3 \\
\hline & E3 II trimester & 0.342 & 0.317 & 0.95 & 60.0 & 34.0 \\
\hline
\end{tabular}

Legend: Bold - significant; MoM - multiples of median; AUC - area under curve; $\beta$ HCG - Beta subunit of human chorionic gonadotropin; PAPPA - pregnancy-associated plasma protein A; AFP - alpha feto protein; E3 - estriol. 


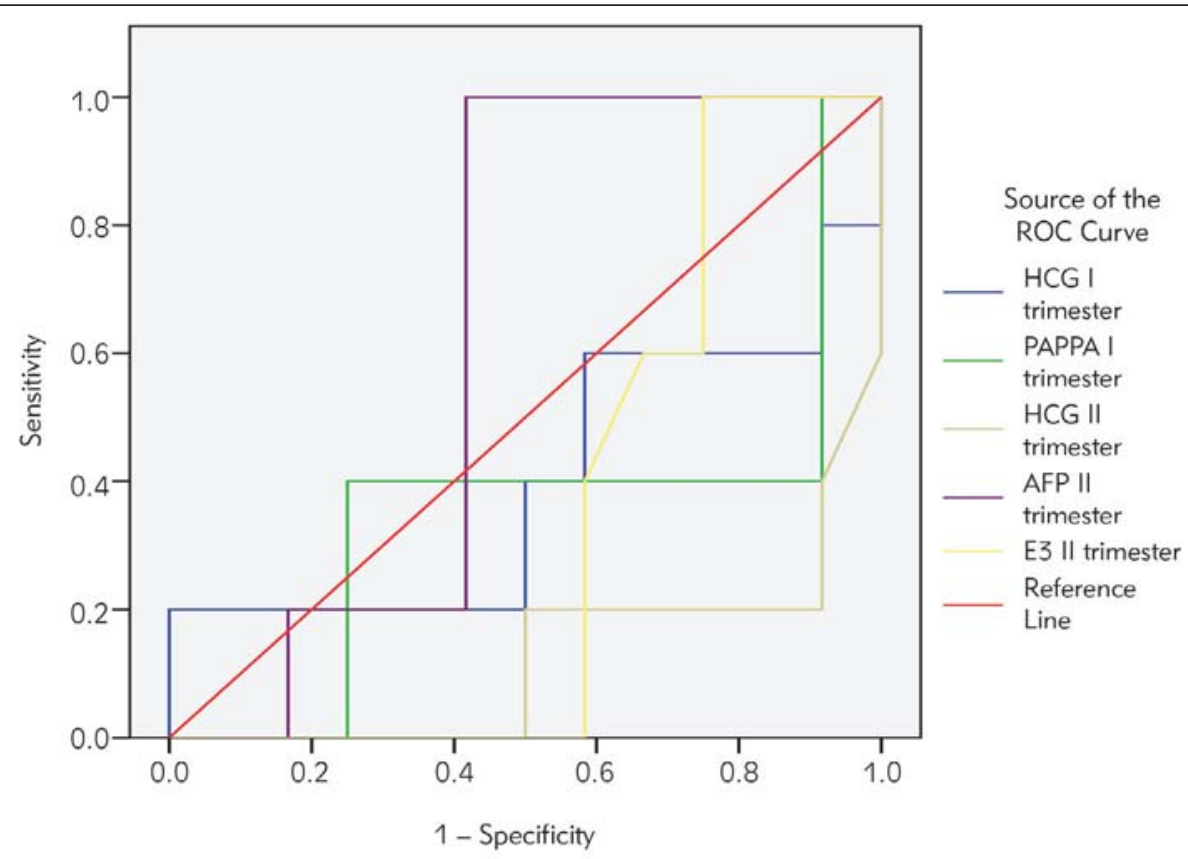

Figure 1 ROC curve for prediction of GH/PE occurrence throughout pregnancy based on biochemical markers (in MoM) of first and second trimester.

Legend: HCG - Beta subunit of human chorionic gonadotropin; PAPPA - pregnancy-associated plasma protein A; AFP - alpha feto protein; E3 - estriol; MoM - multiples of median.

\section{Discussion}

It is well known that the pathophysiology of preeclampsia is based on reduced angiogenesis occurring in early stages of placental formation that causes abnormal placental development and function (13, 14). The defective early trophoblastic invasion of maternal spiral arteries is associated with reduced placental perfusion, endothelial dysfunction and deregulated secretory activity of the trophoblasts. Consequently, placental ischemia leads to release of inflammatory factors, platelet activation, endothelial dysfunction, maternal renal dysfunction or abnormal oxidative stress $(11,15)$. Therefore, it is not surprising that in patients with $\mathrm{GH} / \mathrm{PE}$ numerous biochemical markers, which represent measurable manifestations of impaired placentation, were found to be either decreased or increased in comparison with serum concentrations in physiological pregnancies $(5,14)$. Available literature data show that most changes in serum levels in women with preeclampsia occur for Beta HCG, PAPPA, AFP, estriol and inhibin (15-17). Therefore, it was assumed that measuring these parameters in the first and/or second trimester could be used for prediction of the development of $\mathrm{GH} / \mathrm{PE}$ in later gestational weeks. Moreover, these parameters are widely used for prediction of chromosomal aneuploidies and therefore, these markers are easily accessible for evaluation in our population.

Human chorionic gonadotropin ( $\mathrm{hCG}$ ) is a hormone secreted by syncytiotrophoblast cells. It has two subunits (alpha and beta) out of which beta is hor- mone specific. HCG is necessary for adequate development and function of decidual spiral arteries and in that manner maintaining vascular supply of the pregnancy. Elevated serum concentrations (above $3 \mathrm{MoM}$ ) of HCG in the second trimester have been associated with the development of preeclampsia $(15,18)$.

Pregnancy-associated plasma protein A (PAPPA) is a metalloprotease produced by placental syncytiotrophoblasts. It is involved in the metabolism of insulin-like growth factors and therefore can influence the growth of both placenta and fetus. Decreased serum concentrations of PAPPA (under $1 \mathrm{MoM}$ ) were found in pregnancies complicated with PE or adverse pregnancy outcomes reflecting poor placental function $(13,19)$. However, measurement of PAPP-A alone cannot be used as screening for PE because less than $25 \%$ of affected cases have serum levels below the fifth percentile (11).

Alpha Feto Protein (AFP) is a glycoprotein that is secreted from the yolk sac, fetal liver and gastrointestinal tract. It can be transported to the maternal serum by diffusion across membranes and placenta. Serum levels of AFP increased over 2.5 MoM have been associated in some literature data with developing gestational hypertension and preeclampsia $(15,20)$.

Estriol (E3) during pregnancy is synthesized in very high quantities (100 times higher) by the placenta. In preeclampsia patients, the aberrant synthesis, metabolism, and accumulation of estrogens and estrogen metabolites are registered. Serum concen- 
trations of E3 below 5 percentiles are associated with adverse pregnancy outcomes (21).

Inhibin $\mathrm{A}(\mathrm{INH})$ is also produced by the syncytiotrophoblasts and can be a biochemical marker of placental function. It is involved in the regulation of cell growth and immunologic recognition. In some studies, elevation of inhibin correlated with higher risk of preeclampsia $(15,16,13)$.

Several other biochemical parameters have shown promise for prediction of preeclampsia, such as elevated levels of placental growth factor (PIGF), soluble fms-like tyrosine kinase-1 (sFlt-1), and soluble endoglin (sENG), or decreased concentrations of placental protein 13 (PP13). Still, further studies are needed to determine their actual clinical relevance $(15,8,12)$.

On the other hand, data from current literature are still contradictory regarding the strength of association between pregnancy biochemical markers and $\mathrm{GH} / \mathrm{PE}$ (17). While some studies found significant correlations with numerous markers of both first and second pregnancy trimester with $\mathrm{GH} / \mathrm{PE}(22,23,9)$, other investigations could not confirm these results (24-26). In the majority of studies, among all investigated factors, strong associations were found for only a couple of biochemical markers $(5,19)$. Moreover, time of onset and severity of GH/PE seem to be of major importance when assessing the association of biochemical pregnancy markers with GH/PE development (27). Some authors reported that major decrease in PAPPA and PIGF levels occurs only in the second trimester, implying that measurements of these parameters in the first trimester may not be reliable enough (13). Contrary, in several studies biochemical markers such as Beta HCG and PIGF were significantly correlated only with early and not late onset GH/PE $(5,28)$. According to our study results, serum levels of PAPPA in the first as well as HCG and AFP in the second trimester of pregnancy were significantly higher in women who developed early or late onset GH/PE as well as placental abruption during pregnancy.

Therefore, regression analysis is commonly performed in literature to test more thoroughly the value of biochemical markers for prediction of gestational hypertension development (17). Models from the literature are mostly adjusted for medical history data (Park, Oliveira). For early-onset PE, literature data show that prior PE or chronic HTA, multiparity, BMI, smoking PAPP-A, PIGF, CHTN, PTL and SBP/DBP were confirmed as significant predictors. On the other hand, the predictors of late-onset PE were PAPP-A, PIGF, BMI and prior PE or chronic HTA $(7,29,30$, 31). In some studies, it was determined that the detection rates of single markers for prediction of early-onset GH/PE were relatively low ( $22 \%$ to $83 \%$ ), while for combinations of multiple markers assessed as a prediction model detection rates were significantly higher, between 38\% and reaching even 100\% (32). In prediction models that the authors of this study constructed, Beta HCG, PAPPA and AFP were found to be good predictors of GH/PE occurrence. However, it was determined that different markers should be used for early and late onset GH/PE. This is in accordance with findings from other investigations according to which hemodynamic changes and placental lesions in early and late-onset preeclampsia are diverse and therefore, the biomarkers for their identification should also be different (4).

Biochemical markers detectable in maternal serum are often produced in response to the early developmental insults which precede maternal symptoms and so they are thought to have some predictive value when measured earlier in gestation $(1,10)$. In contrast, bioactive peptide concentrations within the maternal blood often change shortly before disease onset. Therefore, these factors are likely to have strong diagnostic potential but have a somewhat more limited predictive capacity $(4,3)$. Furthermore, although a single biomarker might be strongly associated with a disease, it is unlikely to be detectable in every woman who develops the condition (32). Consequently, when the reliability of biochemical markers as predictors of gestational hypertension was tested by ROC analysis, high specificity and negative predictive value (over 95\%) were obtained in different studies and populations $(29,22)$. However, sensitivities and positive predictive vales were mostly lower (around $70 \%$ ), suggesting limited external validity. Moreover, according to available literature, prediction of early-onset GH/PE based on biochemical markers was easier and more reliable than late-onset changes (2). For inhibin A, studies reported GH/PE detection rates of $35 \%$, while for PAPP-A the detection rates ranged from $22 \%$ to $43 \%$ and were generally higher in early-onset GH/PE. In our study, only AFP was found to significantly predict $\mathrm{GH} / \mathrm{PE}$ late-onset or regardless of its commencement. It explained $63.3 \%$ of cases with sensitivity of $80 \%$. According to literature, AFP could properly predict $77 \%$ of $\mathrm{GH} / \mathrm{PE}$ cases (24). Further studies should clarify how to improve the predictive values of biochemical markers. Majority of authors believe that multivariate screening algorithms could offer the best solution (30-32).

\section{Conclusion}

Effective screening for gestational hypertensive disorders during pregnancy can be achieved by biochemical markers determination in the first and second trimester. However, different markers should be used for early and late onset GH/PE. Further studies should be undertaken to construct the optimal algorithms of biochemical pregnancy markers that could be able to reliable predict GH/PE occurrence.

\section{Conflict of interest statement}

The authors declare that they have no conflict of interest. 


\section{References}

1. Cuffe JSM, Holland O, Salomon C, Rice GE, Perkins AV. Review: Placental derived biomarkers of pregnancy disorders. Placenta 2017; 54:104e110.

2. Ilekis JV, Tsilou E, Fisher S, Abrahams VM, Soares MJ, Cross JC, Zamudio S, Illsley NP, Myatt L, Colvis C, Costantine MM, Haas DM, Sadovsky Y, Weiner C, Rytting $\mathrm{E}$, Bidwell G. Placental origins of adverse pregnancy outcomes: potential molecular targets: an Executive Workshop Summary of the Eunice Kennedy Shriver National Institute of Child Health and Human Development. Am J Obstet Gynecol 2016; 215: S1-S46.

3. Baschat AA, Madger LS, Doyle LE, et al. Prediction of preeclampsia utilizing the first trimester screening examination. Am J Obstet Gynecol 2014; 211: 524.e1-524.e7.

4. Sonek J, Krantz D, Carmichael J, Downing C, Jessup K, Haidar Z, Ho S, Hallahan T, Kliman HJ, McKenna D. First-trimester screening for early and late preeclampsia using maternal characteristics, biomarkers, and estimated placental volume. Am J Obstet Gynecol 2017 doi: 10.1016/j.ajog.2017.10.024.

5. Di Lorenzo G, Ceccarello $M$, Cecotti $V$, Ronfani $L$, Monasta L, Vecchi Brumatti L, Montico M, D'Ottavio G. First trimester maternal serum PIGF, free b-hCG, PAPPA, PP-13, uterine artery Doppler and maternal history for the prediction of preeclampsia. Placenta 2012; 33: 495 e501.

6. Lagana AS, Giordano D, Loddo S, Zoccali G, Vitale SG, Santamaria A, Buemi M, D'Anna R. Decreased Endothelial Progenitor Cells (EPCs) and increased Natural Killer (NK) cells in peripheral blood as possible early markers of preeclampsia: a case-control analysis. Arch Gynecol Obstet 2017; 295: 867-72.

7. Park FJ, Leung CHY, Poon LCY, Williams PF, Rothwell SJ, Hyett JA. Clinical evaluation of a first trimester algorithm predicting the risk of hypertensive disease of pregnancy. ANZJOG 2013; 53: 532-9.

8. Sahai K, Saraswathy S, Yadav TP, Arora D, Krishnan M. Pre-eclampsia: Molecular events to biomarkers. Med J Armed Forces India 2017; 73: 167-74.

9. Paridaens H, Gruson D. Pre-eclampsia: overview on the role of biomarkers in 2016. Ann Biol Clin (Paris) 2017; 75: 245-58.

10. Yliniemi A, Makikallio K, Korpimaki T, Kouru $H$, Marttala J, Ryynanen M. Combination of PAPPA, fhCG $\beta$, AFP, PIGF, sTNFR1, and maternal characteristics in prediction of early-onset preeclampsia. Clin Med Ins: Reprod Health 2015; 9: 13-20.

11. Poon L, Nicolaides K. First-trimester maternal factors and biomarker screening for preeclampsia. Prenatal Diagnosis 2014; 34: 618-27.

12. Anderson UD, Olsson MG, Kristensen $\mathrm{KH}$, Akerstrom B, Hansson SR. Review: biochemical markers to predict preeclampsia. Placenta 2012; 33: S42-47.

13. Lambert-Messerlian G, Eklund EE, Chien EK, RoseneMontella K, Neveux LM, Haddow HRM, Palomaki GE.
Use of first or second trimester serum markers, or both, to predict preeclampsia. Pregnancy Hypertension: Int J Women's Cardiovasc Health 2014; 4: 271-8.

14. Huppertz B, Kawaguchi R. First trimester serum markers to predict preeclampsia. Wien Med Wochenschr 2012; 162: 191-5.

15. Olsen RN, Woelkers D, Dunsmoor-Su R, LaCoursiere DY. Abnormal second-trimester serum analytes are more predictive of preterm preeclampsia. Am J Obstet Gynecol 2012; 207: 228.e1-7.

16. Ree PH, Hahn WB, Chang SW, Jung SH, Kang JH, Cha $\mathrm{DH}$, Kang MS, Huh JY. Early detection of preeclampsia using inhibin a and other second-trimester serum markers. Fetal Diagn Ther 2011; 29: 280-6.

17. Yefet E, Kuzmin O, Schwartz N, Basson F, Nachum Z. Predictive Value of Second-Trimester Biomarkers and Maternal Features for Adverse Pregnancy Outcomes. Fetal Diagn Ther 2017 doi: 10.1159/000458409.

18. Fafula VR, lefremova PU, Onufrovych KO, Maksymyuk VH, Besedina SA, Nakonechnyi Al, Vorobets ZD, Vorobets DZ. Alterations in Arginase-No-Synthase System of Spermatozoa in Human Subjects with Different Fertility Potential. J Med Biochem 2018; 37: 134-40.

19. Kaijomaa M, Rahkonen L, Ulander VM, Hamalainen E, Alfthan H, Markkanen H, Heinonen S, Stefanovic V. Low maternal pregnancy-associated plasma protein $A$ during the first trimester of pregnancy and pregnancy outcomes. Int J Gynecol Obstet 2017; 136: 76-82.

20. Huang T, Hoffman B, Meschino W, Kingdom J, Okun N. Prediction of adverse pregnancy outcomes by combinations of first and second trimester biochemistry markers used in the routine prenatal screening of Down syndrome. Prenat Diagn 2010; 30: 471-7.

21. Jobe SO, Tyler CT, Magness RR. Aberrant synthesis, metabolism and plasma accumulation of circulating estrogens and estrogen metabolites in preeclampsia: implications for vascular dysfunction. Hypertension 2013; 61: 480-7.

22. Cohen JL, Smilen KE, Bianco AT, Moshier EM, Ferrara LA, Stone JL. Predictive value of combined serum biomarkers for adverse pregnancy outcomes. Euro J Obstet Gynecol Reprod Biol 2014; 181: 89-94.

23. Gu W, Lin J, Hou Y. The value of maternal first and second trimester serum data of $\beta$-hCG, PAPP-A, AFP and $\mathrm{uE3}$ in the prediction of preeclampsia. Zhonghua $\mathrm{Fu}$ Chan Ke Za Zhi 2015; 50: 101-7.

24. Nevalainen J, Korpimaki T, Kouru H, Sairanen $M$, Ryynanen M. Performance of first trimester biochemical markers and mean arterial pressure in prediction of early-onset pre-eclampsia. Metabolism Cin Experiment 2017; 175: 6-15.

25. Al-Mukaynizi FB, Alkhuriji A, Babay Z, Addar M, Aldaihan S, Alanazi $M$, Warsy AS. Lack of association between angiotensin converting enzyme $\mathrm{i} / \mathrm{d}$ polymorphism and unexplained recurrent miscarriage in Saudi Arabia. J Med Biochem 2016; 35: 166-73. 
26. Krnjeta T, Mirković Lj, Ignjatović S, Tomašević D, Lukić J, Topalov D, Soldatović I, Majkić-Singh N. Protective role of maternal P.VAL158MET Catechol-O-Methyltransferase polymorphism against early-onset preeclampsia and its complications. J Med Biochem 2016; 35: 312-8.

27. Jelliffe-Pawlowski LL, Baer RJ, Currier RJ, Lyell DJ, Blumenfeld YJ, El-Sayed YY, Shaw GM, Druzin ML. Early-onset severe preeclampsia by first trimester pregnancy-associated plasma protein A and total human chorionic gonadotropin. Am J Perinatol 2015; 32: 703-12.

28. Revankar VM, Narmada L. Assessment of serum $\beta$ hCG and lipid profile in early second trimester as predictors of hypertensive disorders of pregnancy. Int J Gynecol Obstet 2017; 138: 331-4.

29. Oliveira N, Magder LS, Blitzer MG, Baschat AA. Firsttrimester prediction of pre-eclampsia: external validity of algorithms in a prospectively enrolled cohort. Ultrasound Obstet Gynecol 2014; 44: 279-85.

30. Scazzocchio E, Figueras F, Crispi F, Meler E, Masoller N, Mula R, Gratacos E. Performance of a first-trimester screening of preeclampsia in a routine care low-risk setting. Am J Obstet Gynecol 2013; 208:203e. 1-10.

31. Parra Cordero M, Rodrigo R, Barja P, Bosco C, Rencoret G, Sepulveda-Martinez A, Quezada S. Prediction of early and late pre-eclampsia from maternal characteristics, uterine artery Doppler and markers of vasculogenesis during first trimester of pregnancy. Ultrasound Obstet Gynecol 2013; 41: 538-44.

32. Kuc S, Wortelboer EJ, van Rijn BB, Franx A, Visser GH, Schielen PC. Evaluation of 7 serum biomarkers and uterine artery Doppler ultrasound for first-trimester prediction of preeclampsia: a systematic review. Obstet Gynecol Surv 2011; 66: 225-39. 